\author{
Submitted: \\ 17.06.2021 \\ Accepted: \\ 27.08.2021 \\ Published: \\ 29.11 .2021

\section{Twinkle artifact in renal ultrasound, is it a solid point for the diagnosis of renal stone in children?} \\ Moath AlSaiady@, Ahmad Alqatie, Musab Almushayqih \\ Radiology, KFSH \& RC, Saudi Arabia \\ Correspondence: Moath AlSaiady, Radiology, KFSH \& RC, Saudi Arabia; \\ e-mail:msaiady@gmail.com
}
Keywords
twinkle, twinkling, stones, kidneys, ultrasound

DOI: $10.15557 / J \circ U .2021 .0048$

\begin{abstract}
Background: Twinkle artifact, also known as color Doppler comet-tail artifact, occurs behind very strong, granular, and irregular reflecting interfaces such as crystals, stones, or calcification. This is visualized as a random mixture of red and blue pixels in the high-frequency shift spectrum located deep to the interface. Study results have suggested that the sonographic twinkling artifact may aid in the detection of renal stones with a variety of reference standard imaging modalities, including abdominal radiography, excretory urography, gray-scale sonography, and CT. Material and methods: Our retrospective observational study included children who had undergone abdomen/renal ultrasound for kidneys stones in our radiology department between 2013 and 2019. Presence of the twinkle artifact, and stone numbers and sizes were documented. CT examinations done $<3$ months prior to or after US were retrospectively assessed to confirm the presence of kidney stones as a reference standard. Results: Thirty-three abdominal renal US scans of 33 patients ( 21 males, 12 females) fulfilled the entry criteria. The interval between the US and CT was $<3$ months for all patients. The median overall age of the patients was 4 years (IQR: 3.125, range: $1-165$ months), The median number of days between the US and CT was 13 (IQR: 26, range: 0-81 days). US detected 33 hyperechoic foci suspected to be stones; 26 were confirmed as true positive (i.e. showed the twinkle artifact and were seen in CT), 4 were false positive (showed the twinkle artifact but were not seen in CT), and 3 were false negative (did not show the twinkle artifact but were seen in CT). The overall median stone size was $2 \mathrm{~mm}$ in the right kidney, and $5 \mathrm{~mm}$ in the left kidney (IQR: $6,11 \mathrm{~mm}$ ), respectively. Twinkle artifact sensitivity was found to be $89.7 \%$ (95\% CI 39.574\%-90\%). The twinkle artifact was assessed in all true-positive stones, determining a relatively high PPV of 26/29 (86.7\%) for the twinkle artifact. The twinkle artifact was not dependent on stone size. Specificity for the twinkle artifact could not be calculated due to a lack of true negatives. Conclusion: The twinkle artifact is a sensitive US tool for detecting pediatric kidney and ureter stones, but with a small risk of false positive findings.
\end{abstract}

\section{Introduction}

Renal stone is one of the common findings in daily medical practice, and it is usually seen in the adult population rather than the pediatric population ${ }^{(1)}$. However, it has been noticed that the incidence of renal stones has increased 5 times in comparison to the previous decade in the pediatric age group ${ }^{(2)}$.

Renal stones are considered less common in children in comparison to the adult population, and result from a multifactorial process that involves both the patient's underlying metabolic background and environmental conditions promoting nephrolithiasis, such as volume depletion, infection, or the intake of foods high in lithogenic solutes ${ }^{(3,4)}$. In view of the fact that the vast majority of pediatric stones are calcium based ${ }^{(5)}$, investigating causes should be focused on factors that contribute to an increased calcium excretion $^{(4)}$. There are some known underlying metabolic disturbances which increase the risk of stone development, such as Dent disease, primary hyperoxaluria, or Lesch-Nyhan syndrome ${ }^{(4)}$.

There are no typical presenting symptoms of renal stones in the pediatric age group, and they usually present with 
nonspecific poorly localized pain in the abdomen or pelvis $^{(6)}$. Gross hematuria can also be seen in adolescents ${ }^{(4)}$.

A nonenhanced computed tomography (CT) scan is the modality of choice to evaluate renal stones, being characterized by both high sensitivity and specificity. However, the evaluation of renal stones in children should be performed while limiting the ionizing radiation as far as possible. Therefore, ultrasound (US), being a widely available technique, is considered the modality of choice to obtain non-ionizing higher resolution images. According to the recently published guideline, US should be the initial imaging modality for use in children with nonspecific abdominal symptoms and those suspected of having renal stones ${ }^{(7)}$.

Several ultrasonographic features are known to be associated with renal stones, which have been described in the literature, such as the presence of the twinkle artifact. One can identify the twinkle artifact as an intrinsic machine noise which presents as a focus of alternating colors on the Doppler mode signals, related to a strongly reflecting interface of an object and giving the appearance of turbulent blood flow.

A few studies have confirmed the usefulness of the twinkle artifact in detecting renal stones, but with variable degrees of sensitivity. For example, a study performed in an adult age group found that the twinkle artifact was associated with a high sensitivity for detecting renal stones but also with a high false positive rate ${ }^{(8)}$. Another study compared the presence of the renal twinkling artifact in association with nephrolithiasis, revealing that it was relatively insensitive in routine clinical practice and had a high false positive rate $^{(9)}$. On the other hand, some studies mention that the use of color-Doppler US is a preferable option for the sensitive detection of tiny nephrolithiasis ${ }^{(10)}$. One recent study conducted in a pediatric age group showed that the twinkle artifact was characterized by sensitivity reaching up to $88 \%$ $(95 \% \text { CI } 72-96 \%)^{(11)}$.

The purpose of our study was to assess the sensitivity of the twinkle artifact as an aiding tool to diagnose kidney stones in the pediatric age group by ultrasound.

\section{Material and methods}

The study had a retrospective design. Local institutional approval was acquired. The study population consisted of pediatric patients $(<14$ years) who had undergone abdomen/renal ultrasound in a single healthcare facility between February, 2013, and August, 2019. All finalized abdomen/renal ultrasound reports containing the words twinkle, twinkling, and kidney stones $(n=287)$ were identified by query of the institutional picture archiving and communication system (PACS).

Our inclusion criteria were: patient age $<14$ years, availability of images from a reference standard nonenhanced thin-section $(\leq 2.5 \mathrm{~mm})$, and abdominal CT examination performed within 90 days before or after the abdomen/ renal ultrasound examination $(n=33)$.
The exclusion criteria were: any patient who received treatment or intervention for kidney stones such as nephrostomy tubes in between the ultrasound and CT, and any patient with nephrocalcinosis.

Anonymized US and CT reports and images were randomized and retrospectively reviewed by three radiologists (pediatric radiology consultant, pediatric radiology fellow, and senior radiology resident). The following parameters were recorded: gender of patient (male/female), age of patient (months), stone with the twinkle artifact (yes/no), number of stones, location of stones (right/left, kidney), size of stone (mm), is the stone seen in CT? (yes/no), and number of days between the US and CT assessments.

Abdominal CT was used as the reference standard. Subgroup analysis was based on the discrepancies regarding the presence of the twinkle artifact stone between the US and CT. The first group was true positive (US showed the twinkle artifact and a stone was also seen in CT), the second group was false positive (US showed the twinkle artifact but a stone was not seen in CT), and the third group was false negative (US did not show the twinkle artifact but a stone was seen in CT). SPSS V25 was used for data analysis.

\section{Results}

Thirty-three abdomen/renal ultrasound scans of 33 patients (21 males, 12 females) fulfilled the entry criteria. The interval between the US and CT was $<3$ months for all patients. The median overall age was 4 years (IQR: 3.125 , range: $1-165$ months), and the median number of days between the US and CT was 13 (IQR: 26, range: 0-81 days) (Tab. 1). US detected 33 hyperechoic foci suspected for stones; 26 were confirmed true positive (showed the twinkle artifact and stones were seen in CT) (Fig. 1), 4 were false positive (showed the twinkle artifact but no stones were seen in CT) (Fig. 2), and 3 were false negative (did not show the twinkle artifact but stones were seen in CT). The overall median stone size was $2 \mathrm{~mm}$ in the right kidney, and $5 \mathrm{~mm}$ in the left kidney (IQR: 6,11 mm), respectively.

Twinkle artifact sensitivity was found to be $89.7 \%$ (95\% CI $39.574 \%-90 \%$ ), and specificity could not be calculated due to a lack of true negatives (Tab. 2).

The true positive subgroup consisted of $26 / 30$ (kidney) stones. The median stone size was $10 \mathrm{~mm}$ (IQR: $11 \mathrm{~mm}$, range: $2-25 \mathrm{~mm}$ ), and the median number of days between

Tab. 1. Frequency distribution of demographic variables

\begin{tabular}{|c|c|c|}
\hline \multirow{2}{*}{ Gender } & male & $21(63.6 \%)$ \\
\cline { 2 - 3 } & female & $12(36.4 \%)$ \\
\hline \multirow{2}{*}{ Age } & median & 4.00 \\
\cline { 2 - 3 } & IQR & 3.125 \\
\hline \multirow{2}{*}{ Number of stones } & Median & 1.00 \\
\cline { 2 - 3 } & IQR & 1.00 \\
\hline
\end{tabular}


Tab. 2. Subgroups analysis

\begin{tabular}{|c|c|c|c|}
\hline \multicolumn{5}{|c|}{ US findings } \\
\hline & True positive & False positive & False negative \\
\hline Number of cases & 26 & 4 & 3 \\
\hline $\begin{array}{c}\text { Days between } \\
\text { US and CT }\end{array}$ & $(9,23)$ & $(32.5,57.25)$ & $(26,--)$ \\
\hline $\begin{array}{c}\text { \% of twinkle artifact } \\
\text { positive cases }\end{array}$ & $86.7 \%$ & $13.3 \%$ & $\mathrm{~N} / \mathrm{A}$ \\
\hline $\begin{array}{c}\text { Size in mm } \\
\text { (median, IQR) }\end{array}$ & $(10,11)$ & $(8,10)$ & $(15,--)$ \\
\hline
\end{tabular}

the US and CT in this subgroup was 9 (IQR: 23, range: 0-66 days). The twinkle artifact was assessed in all true positive stones, revealing a relatively high PPV of 26/29 $(86.7 \%)$ for the twinkle artifact. The twinkle artifact was not dependent on stone size (Tab. 2).

The false positive subgroup consisted of 4/30 (kidney) foci $(13.3 \%)$. The median stone size was $8 \mathrm{~mm}$ (IQR: $10 \mathrm{~mm}$, range: $3-15 \mathrm{~mm}$ ), and the median number of days between the US and CT in this subgroup was 32.5 (IQR: 57.25, range: $9-81$ days) (Tab. 2).

The false negative subgroup comprised 3 stones (100\%). The median size was $15 \mathrm{~mm}$ (IQR: --, range: $2-15 \mathrm{~mm}$ ), and the median number of days between the US and CT was 26 (IQR: --, range: 11-28 days) (Tab. 2).
There was no difference between the subgroups regarding stone size, age or number of days between the US and CT ( $p=0.767, p=0.736$, and $p=0.194$, respectively).

\section{Discussion}

This was a retrospective observational study using abdominal CT scans as a reference standard to test the hypothesis that the twinkle artifact occurs in most pediatric kidney stones, and with high sensitivity.

The twinkle artifact is a complex phenomenon. The most accepted theory nowadays is that the presence of a narrowband noise due to fluctuations in the circuits of the Doppler US equipment is the underlying cause of this sign. It is mainly observed on rough, hyperechoic, irregular surfaces with multiple cracks which cause a strong reflection of the incident US waves and multiple internal reflections which widen the spectrum. The appearance of the twinkle artifact is correlated with the roughness of the stones - the greater the surface roughness, the greater the artifact ${ }^{(12)}$. This can explain why in 3 cases we did not find the twinkle artifact due to the smooth, regular interface of the stone surface. False positive twinkle artifact is thought to be a result of various causes such as parenchyma-vascular interface, nephrocalcinosis and (to a lesser extent in children) vascular calcification $^{(13)}$.

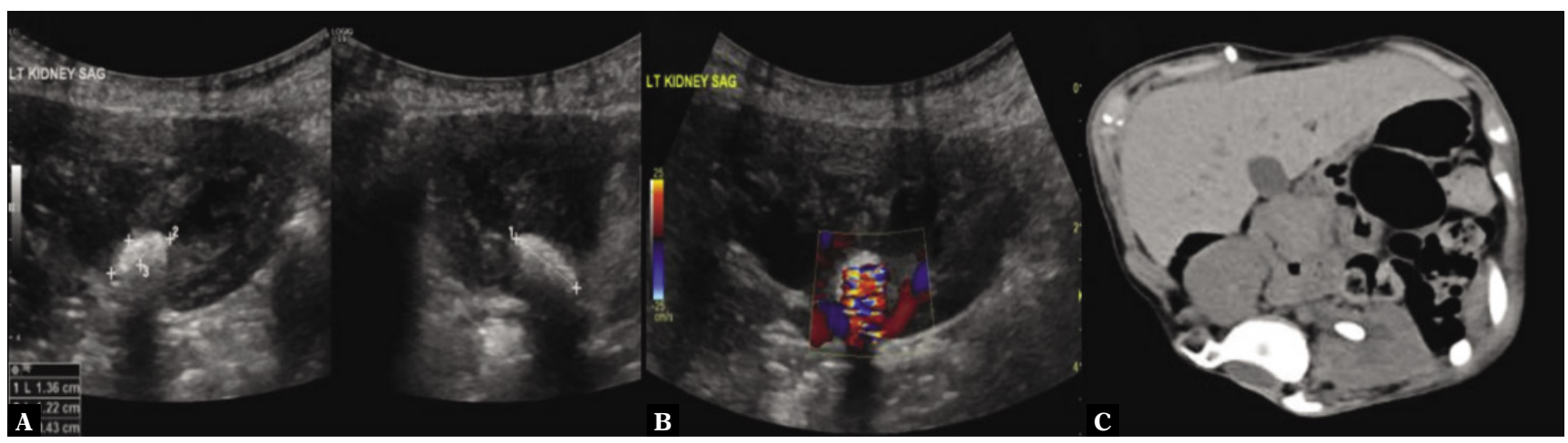

Fig. 1. A 10-year-old female: a known case of thoracolumbar scoliosis. A. Gray-scale ultrasound of the left kidney showed left renal pelvis stone with posterior shadowing, measuring $13.6 \times 12.2 \times 4.3 \mathrm{~mm}$. B. Color-Doppler ultrasound of the left kidney showed the twinkle artifact within the stone. Nonenhanced CT scan of the abdomen and pelvis was done 1 month later, confirming the presence of the stone
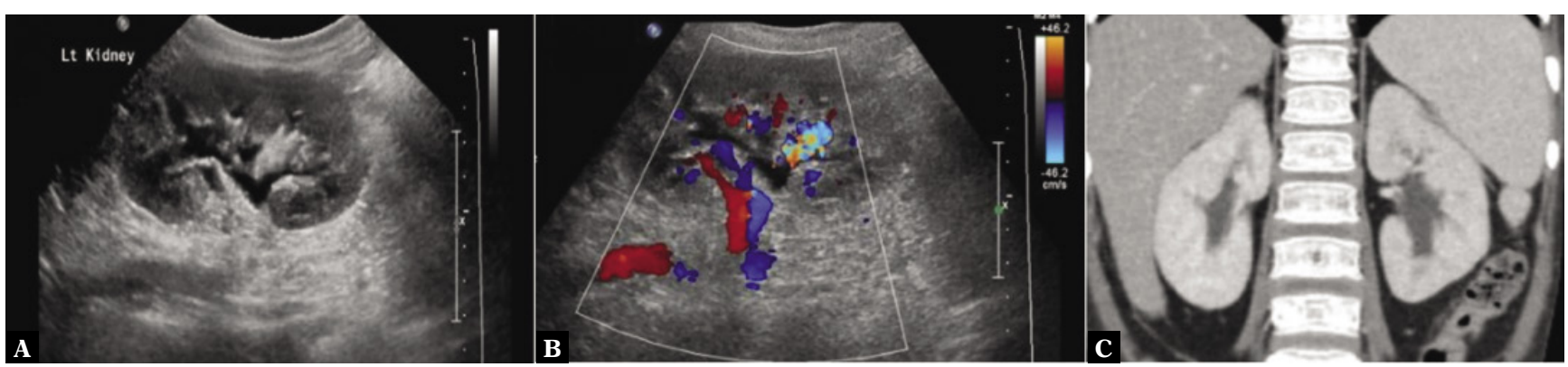

Fig. 2. A 6-year-old male: a known case of acute lymphoblastic leukemia with hematuria. A. Grey-scale ultrasound of the left kidney showed mild left hydronephrosis with non-shadowing echogenic foci in the lower pole. B. Color-Doppler ultrasound of the left kidney showed the twinkle artifact within the left lower pole echogenic focus. Enhanced CT scan of the abdomen and pelvis showed bilateral mild hydronephrosis with no stones in the left kidney 
Our data show high sensitivity of the twinkle artifact in pediatric patients, reaching up to $89.7 \%$ (95\% CI $39.574 \%$ $90 \%$ ), with a relatively high positive predictive value reaching up to $86.7 \%$. However, one needs to take in consideration the fact that there is a small risk of false positive results. Our results are comparable to those obtained in a recent study, showing the sensitivity of $88 \%$ (95\% CI $72-96 \%)^{(11)}$. We found that the twinkle artifact can be detected already in small stones (as small as $2 \mathrm{~mm}$ ).

Although not significant, the median stone size in the true positive subgroup (median: $10 \mathrm{~mm}$ ) was larger than in the false positive subgroup but smaller than in the false negative subgroup median (median values: 8 and $15 \mathrm{~mm}$, respectively).

The limitations of our study include small population size, since we use ultrasound in our center as a primary tool to

\section{References}

1. Issler N, Dufek S, Kleta R, Bockenhauer D, Smeulders N, Van't Hoff W: Epidemiology of paediatric renal stone disease: a 22-year single centre experience in the UK. BMC Nephrol 2017; 18: 136.

2. VanDervoort K, Wiesen J, Frank R, Vento S, Crosby V, Chandra M et al.: Urolithiasis in pediatric patients: a single center study of incidence, clinical presentation and outcome. J Urol 2007; 177: 2300-2305.

3. Coward RJ, Peters CJ, Duffy PG, Corry D, Kellett MJ, Choong S et al.: Epidemiology of paediatric renal stone disease in the UK. Arch Dis Child 2003; 88: 962-965.

4. Sas DJ: An update on the changing epidemiology and metabolic risk factors in pediatric kidney stone disease. Clin J Am Soc Nephrol 2011; 6: 2062-2068.

5. Cameron MA, Sakhaee K, Moe OW: Nephrolithiasis in children. Pediatr Nephrol 2005; 20: 1587-1592.

6. Copelovitch L: Urolithiasis in children medical approach. Pediatr Clin N Am 2012; 59: 881-896.

7. Tasian GE, Pulido JE, Keren R, Dick AW, Setodji CM, Hanley JM et al:: Use of and regional variation in initial CT imaging for kidney stones. Pediatrics 2014; 134: 909-915.

8. Masch WR, Cohan RH, Ellis JH, Dillman JR, Rubin JM, Davenport MS: Clinical effectiveness of prospectively reported sonographic twinkling artifact for the diagnosis of renal calculus in patients without known urolithiasis. Am J Roentgenol 2016; 206: 326-331. detect kidney stones in pediatric patients and reserve CT for complex or questionable cases, if clinically needed; and the lack of knowledge about the biochemical composition of stones which may influence the twinkle $\operatorname{artifact}^{(14)}$.

\section{Conclusion}

The twinkle artifact is a sensitive US tool for detecting pediatric kidney and ureter stones, but with a small risk of false positive findings.

\section{Conflict of interest}

Authors do not report any financial or personal connections with other persons or organizations, which might negatively affect the contents of this publication and/or claim authorship rights to this publication.
9. Dillman JR, Kappil M, Weadock WJ, Rubin JM, Platt JF, DiPietro MA et al: Sonographic twinkling artifact for renal calculus detection: correlation with CT. Radiology 2011; 259: 911-916.

10. Yavuz A, Ceken K, Alimoglu E, Kabaalioglu A: The reliability of color doppler "twinkling" artifact for diagnosing millimetrical nephrolithiasis: comparison with B-Mode US and CT scanning results. J Med Ultrason 2015; 42: 215-222.

11. Verhagen MV, Watson TA, Hickson M, Smeulders N, Humphries PD: Acoustic shadowing in pediatric kidney stone ultrasound: a retrospective study with non-enhanced computed tomography as reference standard. Pediatr Radiol 2019; 49: 777-783.

12. Jamzad A, Setarehdan SK: A novel approach for quantification and analysis of the color Doppler twinkling artifact with application in noninvasive surface roughness characterization: an in vitro phantom study. J Ultrasound Med 2014; 33: 597-610.

13. Rahmouni A, Bargoin R, Herment A, Bargoin N, Vasile N: Color Doppler twinkling artifact in hyperechoic regions. Radiology 1996; 199: 269-271.

14. Gliga ML, Chirila CN, Podeanu DM, Imola T, Voicu SL, Gliga MG et al.: Twinkle, twinkle little stone: an artifact improves the ultrasound performance! Med Ultrason 2017; 19: 272-275. 